\title{
Comparison of Cost Functions for Electrically Driven Running Robots
}

\section{Conference Paper}

Author(s):

Remy, C. David; Buffinton, Keith; Siegwart, Roland

Publication date:

2012

Permanent link:

https://doi.org/10.3929/ethz-a-010023129

Rights / license:

In Copyright - Non-Commercial Use Permitted

Originally published in:

https://doi.org/10.1109/ICRA.2012.6224960 


\title{
Comparison of Cost Functions for Electrically Driven Running Robots
}

\author{
C. David Remy, Keith Buffinton, and Roland Siegwart
}

\begin{abstract}
In this work we apply optimal control to create running gaits for the model of an electrically driven one legged hopper, and compare the results obtained for five different objective functions. By using high compliant series elastic actuators, the motions of joint and motor are decoupled, which allows the exploitation of natural dynamics.

Depending on the cost function, this exploitation varies. Energy is injected at different points of time, the amplitude of actuator action changes significantly, and the optimal gear ratios differ by a factor of two. Variations are, however, comparable over a wide range of hopping heights and running velocities. Purely force-based cost functions prove to be ill-suited for such non-conservative systems, and it is shown that thermal electrical losses, in contrast to common belief, do not dominate energy expenditure. The numerical results are corroborated by detailed analytical considerations which give general insights into optimal excitation with electric actuators.
\end{abstract}

\section{INTRODUCTION}

The exploitation of natural dynamics (i.e., motion induced by inertia, gravity, and elastic oscillations) is one of the most promising ways to improve the energetic efficiency and locomotion speed of legged robots. Through the temporary storage of energy, undesirable negative work can be avoided and surplus energy can be recovered. Actuator action can be spread out over time to decrease peak power, and gears and motors can be protected from impact collisions that inevitably occur when moving at high speed. These principles can be found in biological systems which store energy elastically in muscles and tendons and use the pendulum dynamics of the leg segments for walking and running ([1], [2], [3], [4]), as well as in robotic systems; for example in passive dynamic walkers which create motion exclusively by mechanical dynamics ([5], [6]).

A promising approach to employing these ideas in robotic systems is the use of high compliance series elastic actuation (SEA) [7]. In this design, motors and joints are decoupled by elastic elements that are utilized to periodically store and return energy over the course of the gait cycle and are hence designed as part of the natural dynamics of the system (Fig. 1). The (electric) motors, which act in series, only excite the elastic oscillation and feed energy into the system. Conceptually, this is a major difference to classic series elastic actuators ([8], [9], [10]), in which the spring deflection is regulated to create a well-defined output torque.

Manuscript received September 16, 2011. This research was supported by the Swiss National Science Foundation through the National Centre of Competence in Research Robotics. C. David Remy, and Roland Siegwart are with the Autonomous Systems Lab, Swiss Federal Institute of Technology (ETHZ), Zurich, Switzerland (cremy@ethz.ch, rsiegwart@ethz.ch). Keith Buffinton is with the Department of Mechanical Engineering, Bucknell University, Lewisburg, PA (buffintk@bucknell.edu).

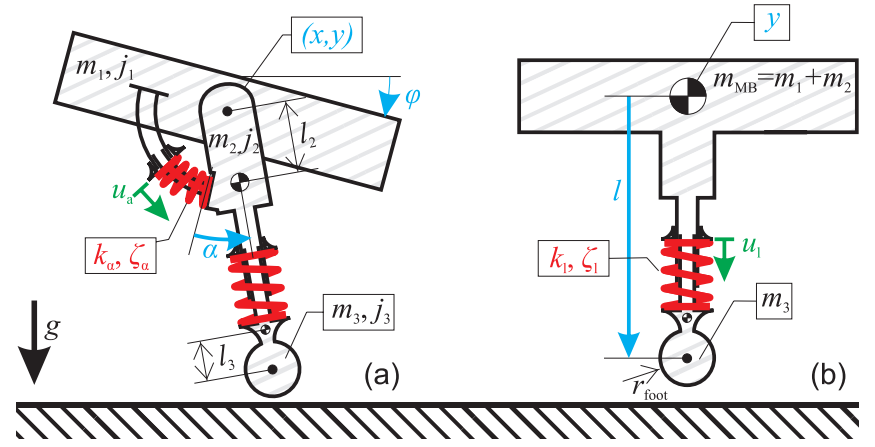

Fig. 1. A planar model of a one legged running robot was used to study different cost functions in gait optimization. All joints were modeled as driven by series elastic actuators. To facilitate analysis, the motion of the model (as shown in a) was initially restricted to in-place hopping (b).

In contrast, actuation is considered to be fully open loop in this paper and generated by the motors following an optimized parametric excitation function.

With the above considerations as background, the primary focus of this work is to compare excitation schemes obtained with a variety of cost functions while using optimal control techniques as tools for gait creation in robotic running. Since the results of every numerical optimization should be treated with great caution, it is an important contribution of this work to compare them to analytical considerations about the structure of optimal solutions.

This idea of using optimization to generate motor inputs for legged systems (both in biology and robotics) has been established for quite some time [11], [12]. See [13] (citations 17-27) for a comprehensive overview. More recent studies by Srinivasan and Ruina (e.g., [14]) examined optimality in legged locomotion with conceptual models that share some similarities with the one presented in this paper. However, in contrast to their investigations (which were aiming at the study of human locomotion) we are particularly interested in robotic systems and thus included a detailed electrical motor model (with force/speed limitations and actuator inertia), damping, and collision losses in our analysis and considered the following cost functions:

- Integral of the square of joint forces

- Thermal electrical losses

- Positive mechanical joint work

- Positive electrical work

- Necessary peak power

In the mathematical formulation of this task, the two requirements for a running gait (periodicity and forward motion) are translated into constraints and define an optimal control 
TABLE I

ALL MODEL PARAMETERS ARE NORMALIZED WITH RESPECT TO TOTAL MASS $m_{o}$, LEG LENGTH $l_{o}$, AND GRAVITY $g$.

$$
\begin{array}{lll}
m_{1}=0.85 m_{o} & j_{3}=0.002 m_{o} l_{o}^{2} & \zeta_{l}=0.2 \\
m_{2}=0.1 m_{o} & l_{2}=0.25 l_{o} & k_{\alpha}=5 m_{o} g l_{o} / \text { rad } \\
m_{3}=0.05 m_{o} & l_{3}=0.25 l_{o} & \zeta_{\alpha}=0.2 \\
j_{1}=0.4 m_{o} l_{o}^{2} & r_{\text {foot }}=0.05 l_{o} & \\
j_{2}=0.002 m_{o} l_{o}^{2} & k_{l}=10 m_{o} g / l_{o} &
\end{array}
$$

problem similar to the formulations of Mombaur [15] and Stelzer [16]. Actuator motion is represented by a parameterized excitation function that makes the problem of finite dimension and suitable for numerical optimization. The study is based on a planar model of a one-legged running robot (similar to the systems in [17]) which can be considered as the archetype of robotic runners. All system parameters, mechanical as well as electrical, are normalized so that results can be applied to a large class of systems [18]. Optimizations were performed with a MATLAB implementation of a direct collocation approach.

\section{METHODS}

\section{A. Model}

The model used in this study has a main body with mass $m_{1}$ and rotational inertia $j_{1}$, an upper leg segment $\left(m_{2}, j_{2}\right)$, and a lower segment $\left(m_{3}, j_{3}\right)$. The position and orientation of the main body is given by $x, y$, and $\varphi$. Main body and upper leg are connected by a rotational hip joint (with joint-angle $\alpha$ ), and the two leg segments are connected prismatically (the resulting leg length $l$ is measured from the hip to the foot-center). The remaining dimensions are shown in Fig. 1. All states and parameters are normalized with respect to total mass $m_{o}$, uncompressed leg length $l_{o}$, and gravity $g$ (Table I). The resting angle of the leg is $\alpha_{o}$.

In the joints, the motion of two adjoining segments is coupled by linear springs with stiffness $k_{l}\left(k_{\alpha}\right)$ and damping ratio $\zeta_{l}\left(\zeta_{\alpha}\right)$. These springs are rigidly attached to the distal segment and, on the other side, connected to an electrical servo-controlled motor with a motor displacement of $u_{l}\left(u_{\alpha}\right)$. Having damping in the springs and a mass associated with the foot means that the system is energetically not conservative (unlike the systems in [14]) and positive net work must be performed by the actuators over the course of a stride.

\section{B. Mechanical Dynamics}

The equations of motion (EoM) are stated in a floating base description as:

$$
\mathbf{M}(\mathbf{q}) \ddot{\mathbf{q}}=\mathbf{h}(\mathbf{q}, \dot{\mathbf{q}})+\mathbf{f}+\mathbf{J}^{T}(\mathbf{q}) \boldsymbol{\lambda}
$$

with the generalized coordinate set $\mathbf{q}=(x y \varphi \alpha l)^{T}$. The dynamics of the system are given by the mass matrix $\mathbf{M}$, the differentiable force vector $\mathbf{h}$ (which includes gravitational and coriolis forces), the actuator forces $\mathbf{f}$, and the contact Jacobian $\mathbf{J}=\partial \mathbf{r} / \partial \mathbf{q}$ which maps a vector of contact forces $\boldsymbol{\lambda}$ into the generalized coordinate space.
During ground contact, the foot is restricted to a pure rolling motion and the contact point vector $\mathbf{r}$ must hold:

$$
\dot{\mathbf{r}}=\mathbf{J} \dot{\mathbf{q}}=\mathbf{0} \text { and } \ddot{\mathbf{r}}=\mathbf{J} \ddot{\mathbf{q}}+\dot{\mathbf{J}} \dot{\mathbf{q}}=\mathbf{0}
$$

Equations (1) and (2) can be solved for the generalized accelerations $\ddot{\mathbf{q}}$ and the contact force $\lambda$. When the normal component of this force becomes negative $\left(\lambda^{y}<0\right)$, the contact opens and the flight phase with $\boldsymbol{\lambda}=\mathbf{0}$ is initiated. At the end of the flight phase $\left(r^{y}=0, \dot{r}^{y}<0\right)$, instantaneous changes in velocities (as a consequence of (2)) lead to external impulsive forces $\boldsymbol{\Lambda}$. To compute these, the equations of motion (1) are integrated over the duration of the collision:

$$
\int_{\left\{t_{o}\right\}}\left\{\mathbf{M} \ddot{\mathbf{q}}-\mathbf{h}-\mathbf{f}-\mathbf{J}^{T} \boldsymbol{\lambda}\right\} d t=\mathbf{M}\left(\dot{\mathbf{q}}^{+}-\dot{\mathbf{q}}^{-}\right)-\mathbf{J}^{T} \boldsymbol{\Lambda}=0
$$

Since the integration is performed over an infinitesimally short time span, the bounded differentiable force vectors $h$ and $\mathbf{f}$ do not contribute and only the impulsive forces and the velocity changes need to be taken into account. Assuming a perfectly inelastic collision [19], the contact point comes to a complete stop after touchdown $\left(\dot{\mathbf{r}}^{+}=0\right)$.

$$
\dot{\mathbf{r}}^{+}=\mathbf{J} \dot{\mathbf{q}}^{+}=\mathbf{J} \mathbf{M}^{-1} \mathbf{J}^{T} \boldsymbol{\Lambda}+\mathbf{J} \dot{\mathbf{q}}^{-}=0
$$

This equation is solved for the collision impulse $\boldsymbol{\Lambda}$ and subsequently for the generalized post impact speeds $\dot{\mathbf{q}}^{+}$.

The mechanical dynamics are driven by the joint forces $\mathbf{f}=\left(\begin{array}{lllll}0 & 0 & 0 & F_{l} & T_{\alpha}\end{array}\right)^{T}$ which are created by the series elastic springs according to:

$$
\begin{array}{r}
F_{l}=k_{l}\left(l_{o}+u_{l}-l\right)+b_{l}\left(\dot{u}_{l}-i\right) \\
T_{\alpha}=k_{\alpha}\left(\alpha_{o}+u_{\alpha}-\alpha\right)+b_{\alpha}\left(\dot{u}_{\alpha}-\dot{\alpha}\right)
\end{array}
$$

The damping coefficients $b_{l}\left(b_{\alpha}\right)$ are computed from the desired damping ratios $\zeta_{l}\left(\zeta_{\alpha}\right)$.

For a large part of this study, the motion is restricted to a single dimension to facilitate analysis and allow analytical predictions with respect to the outcome of the optimization. In these cases, we set $x, \varphi$, and $\alpha$ to zero, and lump the main body and the upper leg together: $m_{m b}=m_{1}+m_{2}$.

\section{Motor Model}

The actuators are modeled as geared DC-motors [20]. Neglecting the electrical dynamics in the inductance of the rotor (which are more than a magnitude faster than the mechanical dynamics), the motor force $F_{l}^{\text {mot }}$ and thermal losses $P_{l}^{\text {loss }}$ (and similarly $T_{\alpha}^{\text {mot }}$ and $P_{\alpha}^{\text {loss }}$ ) are given by

$$
\begin{gathered}
F_{l}^{\text {mot }}=n_{l} k_{l} \frac{1}{R_{l}}\left(U_{l}-\dot{u}_{l} n_{l} k_{l}\right) \\
P_{l}^{\text {loss }}=\frac{1}{R_{l}}\left(U_{l}-\dot{u}_{l} n_{l} k_{l}\right)^{2},
\end{gathered}
$$

with the gear ratio $n_{l}$, the motor constant $k_{l}$, and the armature resistance $R_{l}$. $U_{l}$ is the input voltage that drives the actuator and $\dot{u}_{l}$ is the resulting actuator velocity. No friction is modeled in the actuators, and the maximal noload velocity is thus $\dot{u}_{l}^{\max }=\frac{U_{o}}{n_{l} k_{l}}$, and the maximal motor power $P_{l}^{\max }=\frac{\left(U_{o}\right)^{2}}{R_{l}}$. In order to reduce the number of free parameters in the conceptual models, these quantities of power rating $\left(P_{l}^{\max }\right)$ and power transmission $\left(\dot{u}_{l}^{\max }\right)$ are substituted into eq. (6):

$$
\begin{aligned}
F_{l}^{\text {mot }} & =\frac{P_{l}^{\text {max }}}{\dot{u}_{l}^{\text {max }}}\left(\frac{U_{l}}{U_{o}}-\frac{\dot{u}_{l}}{\dot{u}_{l}^{\text {max }}}\right) \\
P_{l}^{\text {loss }} & =P_{l}^{\text {max }}\left(\frac{U_{l}}{U_{o}}-\frac{\dot{u}_{l}}{\dot{u}_{l}^{\text {max }}}\right)^{2}=\left(F_{l}^{\text {mot }}\right)^{2} \frac{\left(\dot{u}_{l}^{\text {max }}\right)^{2}}{P_{l}^{\text {max }}} .
\end{aligned}
$$


The quotient $\frac{U_{l}}{U_{o}}$ can be interpreted as an electrical normalization, similar to the mechanical normalization by $m_{o}, l_{o}$, and $g$. It is intrinsically limited to \pm 1 .

Thermal losses must be bounded to prevent overheating of the motor. We introduce a thermal loss conversion factor $c_{l}^{l i m}$ that describes the ratio of the maximal admissible current $i_{l}^{\text {lim }}$ to the stall current $c_{l}^{\text {lim }}=i_{l}^{\text {lim }} / \frac{U_{o}}{R_{l}}$ (and hence $P_{l}^{\text {loss }} \leq P_{l}^{\max } \cdot\left(c_{l}^{\text {lim }}\right)^{2}$ ) and state the following conservative limits for motor force and speed:

$$
\left|F_{l}^{\text {mot }}\right| \leq c_{l}^{l i m} \frac{P_{l}^{\text {max }}}{\dot{u}_{l}^{\text {max }}}, \quad\left|\dot{u}_{l}\right| \leq \dot{u}_{l}^{\text {max }}\left(1-c_{l}^{l i m}\right) .
$$

Finally, we have to take into account that the force generated in the motor is the sum of the spring force and the force to accelerate the actuator itself. This is expressed by

$$
F_{l}^{\text {mot }}=F_{l}+\ddot{u}_{l} \frac{j_{l}^{\text {unsc. }}}{\left(\dot{u}_{l}^{\text {max }}\right)^{2}}
$$

with the unscaled inertia $j_{l}^{\text {unsc. }}=j_{l}^{\text {mot }}\left(\dot{u}_{l}^{\max }\right)^{2}$, which transforms the (reflected) motor inertia $j_{l}^{\text {mot }}$ into our normalized framework. In this way, we can reduce all important motor equations to only three free parameters.

\section{Motor Parameters}

To obtain realistic motor parameters, data from 132 motors from the Maxon RE and EC series were evaluated for maximal power $P^{\max }$, the thermal loss conversion factor $c^{l i m}$, and the unscaled inertia $j^{\text {unsc. }}$. Logarithmic regression was performed with respect to the actuator mass $m_{m o t}$ and established that maximal power scaled with $P^{\max }=4700\left(m_{m o t}\right)^{1.35} \mathrm{~W}$, unscaled inertia with $j^{\text {unsc. }}=23.4\left(m_{m o t}\right)^{1.26} \mathrm{~J}$, and admitable losses with $c^{l i m}=0.055\left(m_{m o t}\right)^{-0.37}$. These values differ slightly from those reported in [21].

Since normalized power is expressed in units of $m_{o} g \sqrt{g l_{o}}$, the absolute power requirements of differently sized robots vary greatly, even if the structure and motion in the normalized representation are equal. Yet, to evaluate different cost functions within a normalized framework, a relative comparison is sufficient. To this end, the effective actuator mass of an initial configuration is scaled with a dimensionless scaling parameter $\rho^{m o t}$. We based our analysis on a prototype [22] with a mass of $5 \mathrm{~kg}$ and a leg length of $0.4 \mathrm{~m}$. The total mass of the motors is $0.6 \mathrm{~kg}$. This leads to

$$
\begin{aligned}
P^{\max } & \approx 24\left(\rho^{\text {mot }}\right)^{1.35} \mathrm{~m}_{\circ} \mathrm{g} \sqrt{\mathrm{gl}_{\mathrm{o}}} \\
j^{\text {unsc. }} & \approx 0.6\left(\rho^{\text {mot }}\right)^{1.26} \mathrm{~m}_{\circ} \mathrm{gl} \mathrm{l}_{\mathrm{o}} \\
c^{\text {lim }} & \approx 0.07\left(\rho^{\text {mot }}\right)^{-0.37}
\end{aligned}
$$

\section{E. Excitation}

The motion of the actuators $\mathbf{u}$ is defined by a Fourier series according to:

$$
\mathbf{u}=\sum_{i} \mathbf{a}_{i} \sin (i 2 \pi f t)+\mathbf{b}_{i} \cos (i 2 \pi f t) .
$$

The coefficients $\mathbf{a}_{i}$ and $\mathbf{b}_{i}$ as well as the excitation frequency $f=1 / T$ are the design variables of this representation: $\mathbf{s}=\left(f \mathbf{a}_{i} \mathbf{b}_{i}\right)^{T}$. In contrast to other parametric functions (such as splines, polynomials, or piecewise linear/constant functions), the use of a Fourier series ensures that the activation function is periodic and (by limiting the number of terms in the series) does not exceed the closed loop position control bandwidth of the servo motors. Moreover, the root search and optimization can be initially performed for a small number of terms and successively expanded afterwards.

Within this framework, gait synthesis is considered as the search for initial model states $\mathbf{q}\left(t_{o}\right), \dot{\mathbf{q}}\left(t_{o}\right)$ and excitation parameters $f, \mathbf{a}_{i}, \mathbf{b}_{i}$ that generate a periodic motion. Only $x$ is allowed to be aperiodic, reflecting the desired forward motion. With these two requirements fulfilled, the model is able to perform a continuous forward motion, or in other words, it exhibits a steady gait. With the additional requirement of minimizing the cost function

$$
c=c\left(T, \mathbf{a}_{i}, \mathbf{b}_{i}, \mathbf{q}\left(t_{o}\right), \dot{\mathbf{q}}\left(t_{o}\right)\right)
$$

we transform the search for a periodic hopping/running motion into the constrained optimization problem:

$$
\begin{array}{r}
\min \left\{c\left(f, \mathbf{a}_{i}, \mathbf{b}_{i}, \mathbf{q}\left(t_{o}\right), \dot{\mathbf{q}}\left(t_{o}\right)\right)\right\} \\
\text { s.t. } \quad \mathbf{q}(T)=\mathbf{q}\left(t_{o}\right) \\
\dot{\mathbf{q}}(T)=\dot{\mathbf{q}}\left(t_{o}\right)
\end{array}
$$

\section{F. Optimization}

The optimization problem in (13) was solved by direct collocation [23]. The state variables were discretized on a grid with respect to time and for each grid point a secondorder numerical integration law was used. This transformed equations (1)-(5) into a number of additional constraints [24], which were all solved in parallel. This eliminated the need for numerical integration and greatly improved convergence. The algorithm was implemented [25] and solved numerically using the MATLAB optimization toolbox.

\section{1D ANALYSIS}

As an initial analysis, we compared different cost functions for in-place hopping with the 1D model. The cost functions consequently only include terms for leg extension. We required a hopping height of 1.2, and composed the excitation function of 5 pairs of Fourier terms. $\rho^{\text {mot }}$ was initially set to 1 , which provided ample power resources. As initial seed for the optimization, we simply dropped the model from the desired hopping height with all actuators at rest. All plots in this section show trajectories over a full stride, starting and finishing at apex-transit. Vertical bars indicate the instances of touch-down and lift-off.

\section{A. Joint force}

One of the most commonly used objective functions in the generation of motor inputs for robotics is the integral of the square of actuator torques. We included this objective in two variations. Firstly, we simply integrated the square of the leg spring force as given by eq. (5),

$$
c_{F^{2}}=\int_{0}^{T}\left(F_{l}\right)^{2} d t .
$$

It becomes quickly evident, however, that this objective function is not excessively useful: to spread out the joint force $F_{l}$ over the full stride, the optimizer creates a gait in which the foot is rapidly accelerated towards the ground 
during swing. This generates a reaction force on the main body, and transfers a large fraction of the momentum induced by gravity to the foot where it is 'eliminated' in the ground contact collision. For our model (where only $5 \%$ of the total mass is attributed to the foot) $83 \%$ of the total momentum was generated this way. While this strategy generates a mathematically consistent solution, it produces many undesirable characteristics such as very large actuator accelerations and high energetic losses at the ground contact collision. This makes $c_{F^{2}}$ unsuitable as a cost function.

\section{B. Electrical losses}

We consequently expanded the objective function to include the forces necessary to overcome the inertia of the actuator itself. With proper scaling (as given by (7)), these forces can be mapped into a measure of the thermal losses in the electric motor:

$$
c_{\text {loss }}=\frac{\left(\dot{u}_{l}^{\max }\right)^{2}}{P_{l}^{\max }} \int_{0}^{T}\left(F_{l}^{\text {mot }}\right)^{2} d t .
$$

The optimization must thus be extended to include the transmission ratio $\dot{u}_{l}^{\max }$ as an additional free parameter, whereas $P_{l}^{\max }, c_{l}^{\text {lim }}$, and $j_{l}^{\text {unsc. }}$ are considered to be fixed. It has been argued (e.g., [13], § 15) that employing a series elastic element will not reduce the value of a purely forcebased cost function; a claim that does not hold if an actuator with inertia is included in the analysis. In this case, the forces in motor and spring (as given by (9)) are not equal and it is shown in the following how this is exploited in the excitation.

Since no net momentum is transferred to the actuator inertia or the main body over the course of a full stride, the impulse balance is given as $\int_{0}^{T} F_{l}^{m o t} d t=m_{m b} g \cdot T=$ $\bar{F}_{l}^{\text {mot }} \cdot T$. With this we decompose the motor force into two terms: a constant term that compensates for the gravitational forces and an active term that is used to feed energy into the system: $F_{l}^{m o t}=\bar{F}_{l}^{m o t}+\tilde{F}_{l}^{m o t}$. The motion of the actuator is consequently a superposition of a passive motion induced by the difference between the leg spring force $F_{l}$ and the constant motor force $\bar{F}_{l}^{\text {mot }}$, as well as an active motion induced by the active motor force $\tilde{F}_{l}^{\text {mot }} ; \ddot{u}_{l}=\ddot{u}_{l}^{\text {pas }}+\ddot{u}_{l}^{\text {act }}$ :

$$
\begin{aligned}
& \ddot{u}_{l}^{\text {pas }}=\left(\bar{F}_{l}^{\text {mot }}-F_{l}\right) \frac{\left(\dot{u}_{l}^{\text {max }}\right)^{2}}{j_{l}^{\text {unsc. }}} \\
& \ddot{u}_{l}^{\text {act }}=\tilde{F}_{l}^{\text {mot }} \frac{\left(\dot{u}_{l}^{\text {max }}\right)^{2}}{j_{l}^{\text {unsc. }}} .
\end{aligned}
$$

We use the terms 'active' and 'passive' in this context, since a constant actuator force will not create any net work over the course of a stride. Energy is transferred between the inertia of the actuator and the joint, and alternates between positive joint power and negative joint power, but when integrated over a full stride, the net work evaluates to zero (Fig. 2). Since positive net work is needed to compensate for energetic losses, the active acceleration must extend the leg during the stance phase and retract it during flight. With these considerations, the thermal losses can be expressed as:

$$
\begin{aligned}
c_{\text {loss }} & =\frac{\left(\dot{u}_{l}^{\text {max }}\right)^{2}}{P_{l}^{\text {max }}} \int_{0}^{T}\left(\bar{F}_{l}^{\text {mot }}+\tilde{F}_{l}^{\text {mot }}\right)^{2} d t= \\
& =\frac{\left(\dot{u}_{l}^{\text {max }}\right)^{2}}{P_{l}^{\text {max }}} \int_{0}^{T}\left(m_{m b} g\right)^{2}+2 m_{m b} g \tilde{F}_{l}^{\text {mot }}+\left(\tilde{F}_{l}^{\text {mot }}\right)^{2} d t
\end{aligned}
$$

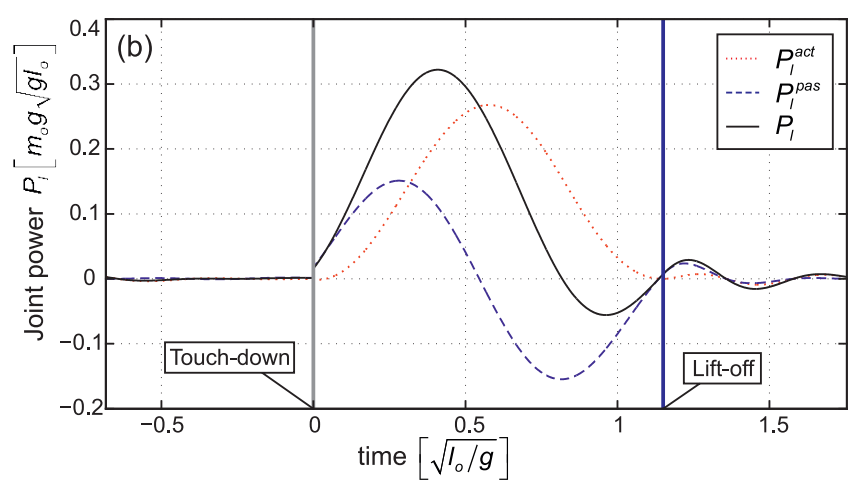

Fig. 2. Actuator acceleration can be decomposed into a passive part $\ddot{u}_{l}^{\text {pas }}$ (induced by the spring force $F_{l}$ and a constant motor force $\bar{F}_{l}^{\text {mot }}$ ), and an active part $\ddot{u}_{l}^{a c t}$ (induced by the 'active' force $\tilde{F}_{l}^{\text {mot }}$ ). As the passive acceleration does not create any net work over the course of a stride, active acceleration becomes necessary to compensate for energetic losses.

Since the active actuator acceleration and thus the active force $F_{l}^{\text {mot }}$ must be balanced over the course of one stride, their integral is equal to zero and we can further simplify the expression to obtain:

$$
\begin{aligned}
c_{\text {loss }} & =\frac{\left(\dot{u}_{l}^{\max }\right)^{2}}{P_{l}^{\max }}\left(\left(m_{m b} g\right)^{2} T+0+\int_{0}^{T}\left(\tilde{F}_{l}^{\text {mot }}\right)^{2} d t\right)= \\
& =\frac{T}{P_{l}^{\max }}\left(\left(m_{m b} g \dot{u}_{l}^{\text {max }}\right)^{2}+\left(\frac{j_{l}^{\text {unsc. }}}{\dot{u}_{l}^{\text {max }}}\right)^{2} \sigma^{2}\left(\ddot{u}_{l}^{a c t}\right)\right)
\end{aligned}
$$

with an optimal transmission ratio of

$$
\dot{u}_{l}^{\max }=\sqrt{\frac{j_{l}^{u n s c .} \sigma\left(\ddot{u}_{l}^{a c t}\right)}{m_{m b} g}},
$$

and thus:

$$
c_{l o s s}=2 T m_{m b} g \frac{j_{l}^{u n s c .}}{P_{l}^{\max }} \sigma\left(\ddot{u}_{l}^{a c t}\right) .
$$

In terms of efficiency, this allows for the following conclusions: this cost function implicitly reduces energetic losses in the system, since they would require compensation through a 'costly' active acceleration $\ddot{u}_{l}^{a c t}$. An undesired amplification of the contact collision as seen for the previous cost function is thus not observed. Negative mechanical work, on the other hand, is not necessarily avoided since energetic fluctuations induced by the passive acceleration $\ddot{u}_{l}^{\text {pas }}$ do not contribute to the overall costs. Equation (18) also shows that electrical thermal losses are directly proportional to the inertia of the actuators used and inversely proportional to the maximal actuator power. These two quantities scale very similarly with the size of the actuator, such that the electrical losses are rather independent of the size of the actuators used.

When trying to minimize $\sigma^{2}\left(\ddot{u}_{l}^{a c t}\right)$, while moving the actuator $u_{l}^{a c t}$ in order to inject the required energy, the optimal solution would be ramping $\ddot{u}_{l}^{a c t}$ linearly between touchdown and lift-off. To some degree, this shape is approximated in the active acceleration shown in Figure 3. However, since the actual motion should also minimize mechanical losses, must be generated by the limited number of Fourier terms, and since the energy injection depends also on the spring 


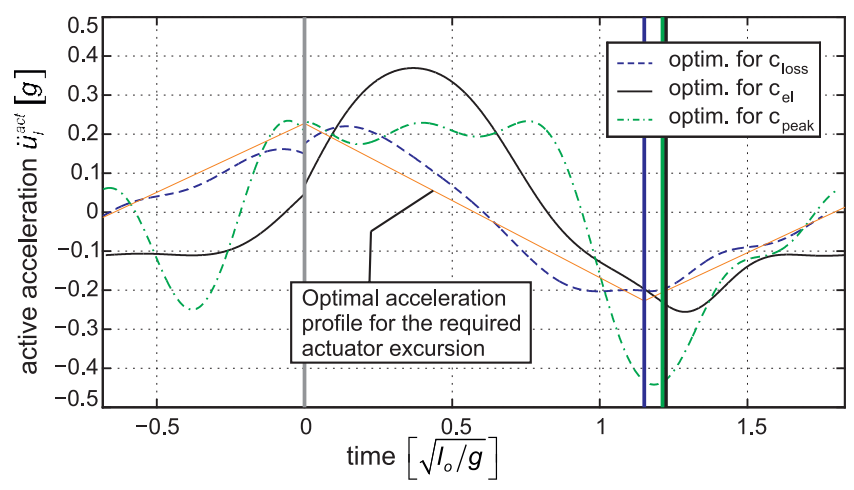

Fig. 3. Active accelerations $\ddot{u}_{l}^{a c t}$ are compared for actuator profiles optimized for electrical losses, electrical work, and peak power. When minimizing the electrical losses $c_{l o s s}$, the actuator is merely used to inject energy during stance, i.e., to move the actuator against the spring. It thus follows roughly the optimal acceleration profile of a point mass. This motion is altered to prevent negative actuator work and reduce damping and collision losses when optimizing the positive electrical work $c_{e l}$ or to reduce the actuator power in order to minimize $c_{\text {peak }}$.

force $F_{l}$, the motion deviates to some degree from linear trajectories.

\section{Positive mechanical joint work}

Mechanical actuator work (the complement of thermal losses) can be stated as:

$$
c_{\text {mech }}=\int_{0}^{T} \max \left(F_{l} \dot{u}_{l}, 0\right) d t .
$$

Reducing the amount of positive work that is performed in the joint implicitly means minimizing:

- The energetic losses in the contact collisions

- Viscous damping in the spring

- Any negative work of the actuator

Touchdown losses can be avoided by reducing the impact speed of the foot, i.e., by retracting the leg shortly before the collision. The overall savings potential of this is rather small and active retraction might actually increase other losses. Damping is caused by the relative motion of the joint and the actuator, and can be expressed in terms of power as $P_{\text {damp }}=b_{l}\left(i-\dot{u}_{l}\right)^{2}$. Damping losses can never be avoided entirely, as the spring must undergo a full compressionextension cycle during stance. Nevertheless, by trying to keep the compression speed $\left(i-\dot{u}_{l}\right)$ constant, the integral of the damping power can be reduced. Finally, to avoid negative actuator work, the actuator must not be retracted $\left(\dot{u}_{l} \geq 0\right)$ during stance when the spring force is positive.

In combination, this means that the actuator remains motionless for the first half of stance $\left(\dot{u}_{l} \approx 0\right)$ to avoid negative work and keep $P_{d a m p}$ small. Throughout the second half of stance, when the leg is extending, the actuator is extending as well, trying to keep $\left(i-\dot{u}_{l}\right)$ constant and thus the integral over $P_{\text {damp }}$ small. In Fig. 4a the actuator profiles optimized for $c_{m e c h}$ and $c_{\text {loss }}$ can be compared. While the actuator motion is homogeneously distributed over the full stride to reduce $\sigma\left(\ddot{u}_{l}^{\text {act }}\right)$ when optimizing $c_{\text {loss }}$, it

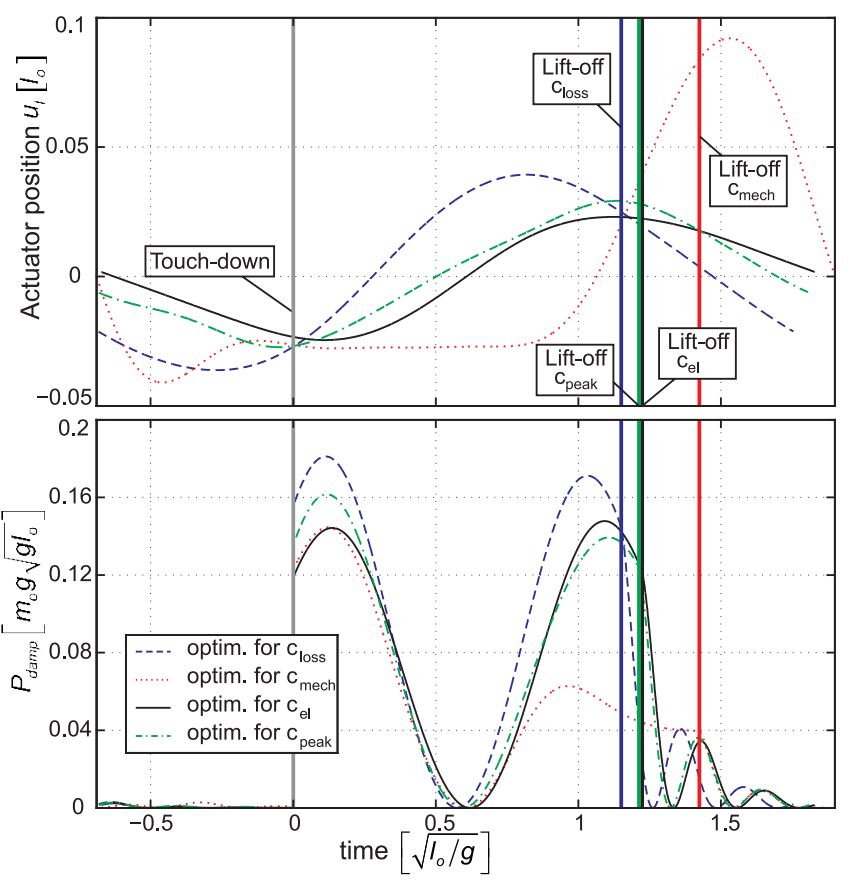

Fig. 4. When optimizing for minimal thermal losses $c_{\text {loss }}$, the actuator motion (a) is homogenously distributed over the full stride. This keeps the active motion of the actuator $\ddot{u}_{l}^{a c t}$ small. In contrast, the actuator remains motionless during the first half of the stance phase and extends rapidly during the second half when trying to minimize the positive mechanical work $c_{m e c h}$. This reduces the damping losses in the spring (b).

remains motionless during the first half of the stance phase for optimization of $c_{\text {mech }}$. Although the subsequent motion is more pronounced, as the same amount of energy must be introduced in less time, the corresponding damping loss $P_{d a m p}$ is greatly reduced in comparison to optimizations with other cost functions (Fig. 4b), especially in the second half of stance.

\section{Positive electrical power}

The most sophisticated cost function based on energetics seeks to directly measure the integral of the positive electrical power of the actuator:

$$
\begin{aligned}
c_{e l} & =\int_{0}^{T} \max \left(F_{l}^{\text {mot }} \dot{u}_{l}+P_{l}^{\text {loss }}, 0\right) d t= \\
& =\int_{0}^{T} \max \left(F_{l}^{\text {mot }} \dot{u}_{l}+\left(F_{l}^{\text {mot }}\right)^{2} \frac{\left(\dot{u}_{l}^{\text {max }}\right)^{2}}{P_{l}^{\text {max }}}, 0\right) d t
\end{aligned}
$$

Apart from transmission losses and inaccuracies in the model, this cost function reflects the actual electrical energy one must provide to keep the robot hopping, assuming that we are unable to recover negative electrical work and store it for later use (which is the case for most of todays robots). When looking at the numerical results (Fig. $4 \& 5$ ), it becomes evident that this function essentially results in a trade-off between optimizations for $c_{\text {loss }}$ and $c_{m e c h}$. For example, the excursion of the actuator is shifted towards the end of stance, yet the motor never really remains motionless as it is the case for $c_{m e c h}$ (Fig. 4a). As a result, the damping losses are smaller than those for profiles optimized 


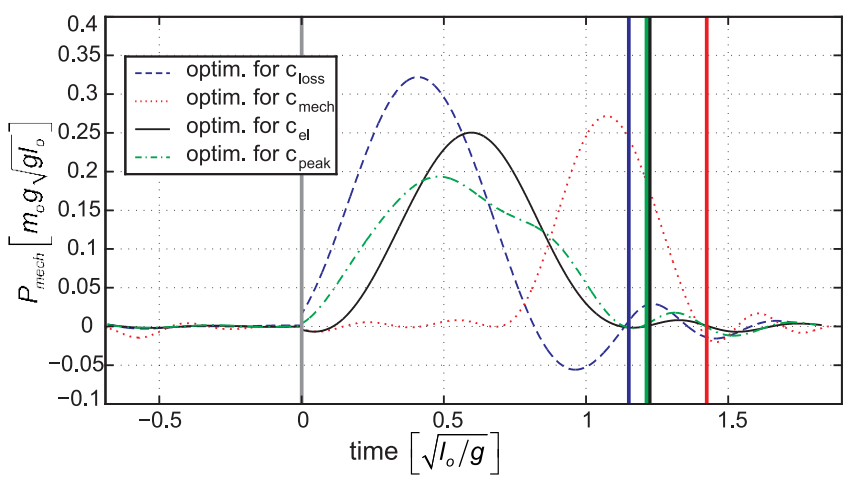

Fig. 5. Mechanical energy is injected early in the stance phase when optimizing for $c_{\text {loss }}$. To a large degree, this is based on a passive energy transfer from the rotor inertia to the joint. This passive transfer is reversed in the second half of stance, where some energy is actually removed from the joint. Negative actuator power is fully avoided when trying to minimize the positive mechanical work $c_{m e c h}$. Optimizing for positive electrical work $c_{e l}$ is a trade-off between these two strategies, where energy is injected in mid-stance. Power is spread out more evenly over the stance phase when minimizing the required actuator size $c_{\text {peak }}$.

for electrical losses but the large reduction in the expansion phase of the spring cannot be observed (Fig. 4b). The same trade-off can be observed for energy injection (Fig. 5), which happens in early stance when the motion is optimized for $c_{\text {loss }}$ (driven by the passive energy transfer from the rotor inertia to the joint), and in late stance when the motion is optimized for $c_{m e c h}$ (to keep the damping losses small). Actuator motion profiles that minimize the positive electrical work $c_{e l}$ injected energy right in the middle of stance. In Fig. 5 one can also observe that negative actuator work is being avoided, since the actuator is always extending while the leg is in ground contact.

Since the performed mechanical work is independent from the gear, $\dot{u}_{l}^{\max }$ can still be computed by (17). In contrast to $c_{\text {loss }}$, the active accelerations $\ddot{u}_{l}^{a c t}$ are now not only used to create a positive net work during stance, but also seek to avoid negative mechanical work and reduce damping and collision losses. A considerable deviation from the theoretically optimal acceleration and those obtained when optimizing for $c_{l o s s}$ can thus be observed in Fig. 3.

\section{E. Actuator mass}

Finally, we examined the excitation inputs that would minimize the mass of the required actuator. With the scaling laws presented earlier, this simply means minimizing the dimensionless scaling parameter $\rho^{\text {mot }}$ as defined in (10). The associated cost function is thus:

$$
c_{\text {peak }}=\rho^{m o t} \text {. }
$$

The optimization problem in (13) must now include the limits for motor force and motor velocity (as they are given by (8)) as additional constraints. To keep peak power small, the mechanical motor power is distributed more equally over the entire stance phase (Fig. 5). The actuator is extended and retracted very steadily (Fig. 4a) and active accelerations during stance are bounded (Figure 3). As a result, the motor scaling parameter can be reduced to $\rho^{\text {mot }}=0.145$. To put this value into context: trajectories that have been optimized for positive electrical work would require a minimal scaling factor of $\rho^{m o t}=0.681$, i.e. 4.7 times as high. The implications of this are discussed in more detail in the conclusions of this paper.

In comparison to the other cost functions, the convergence behavior of $p_{\text {peak }}$ was rather poor. This is obvious, since we were minimizing a variable that acts directly upon the constraints. The cost function becomes highly nonlinear and is not even continuous when constraints are active at multiple locations along the trajectory. Because of this, one can see that $c_{\text {peak }}$ behaves more erratically than the other objective functions, when costs are evaluated as a function of a changing parameter (as can be seen later on in Figures 8a).

\section{2D ANALYSIS}

For the 2D analysis, we studied forward running with a normalized velocity of $\dot{x}=1$. Hopping height was set at 1.2 times the leg length to obtain results comparable with the 1D analysis. Five pairs of Fourier terms were included in the optimization and the motor model and cost functions introduced in the previous sections were extended to include hip actuation, as well. Because of the poor convergence behavior of $c_{\text {peak }}$, we limited the analysis to the three cost functions $c_{l o s s}, c_{m e c h}$, and $c_{e l}$. All cost functions were evaluated for a full step. To avoid the singularity at $\dot{x}=$ 0 , costs were not normalized with respect to the distance traveled (i.e., as a measure of cost of transportation (COT) [26]) but evaluated per hop. This allowed the 2D optimization to build directly upon the $1 \mathrm{D}$ results. Since the forward velocity (at least for the air phase) was fixed, the distinction between the two approaches is not too critical.

The excitation profiles for leg extension were qualitatively identical to those obtained for in-place hopping (Fig. 6a). Yet, the mechanical losses and thus the necessary actuator action were almost doubled. Since the leg hit the ground with an angle of attack of about $30^{\circ}$, the horizontal motion of the runner had a substantial impact on the prismatic action of the leg. For example, to accommodate the horizontal component of the main body's velocity, leg compression speed was increased by up to $50 \%$. This doubled the damping losses in the system which accounted for the major part of the mechanical losses.

For hip swing actuation, two principally different results were observed. When optimized for $c_{m e c h}$, and $c_{e l}$, the SEAs were essentially used as force controllable actuators. No energy was stored in the hip spring, and it was only compressed when a torque was needed to reverse the direction of motion of the swinging leg (shortly before touch-down and shortly after lift-off). For the remainder of the stride, the actuator followed the motion of the leg without producing any torque at all. Because of this, damping losses in the spring were almost nonexistent. To follow the natural pendulum dynamics of the leg, the motor needed to be fast while producing relatively small torques. Consequently, a low gear reduction was chosen by the optimizer. Since elastic energy storage was not exploited, the only benefits of the compliant actuators 


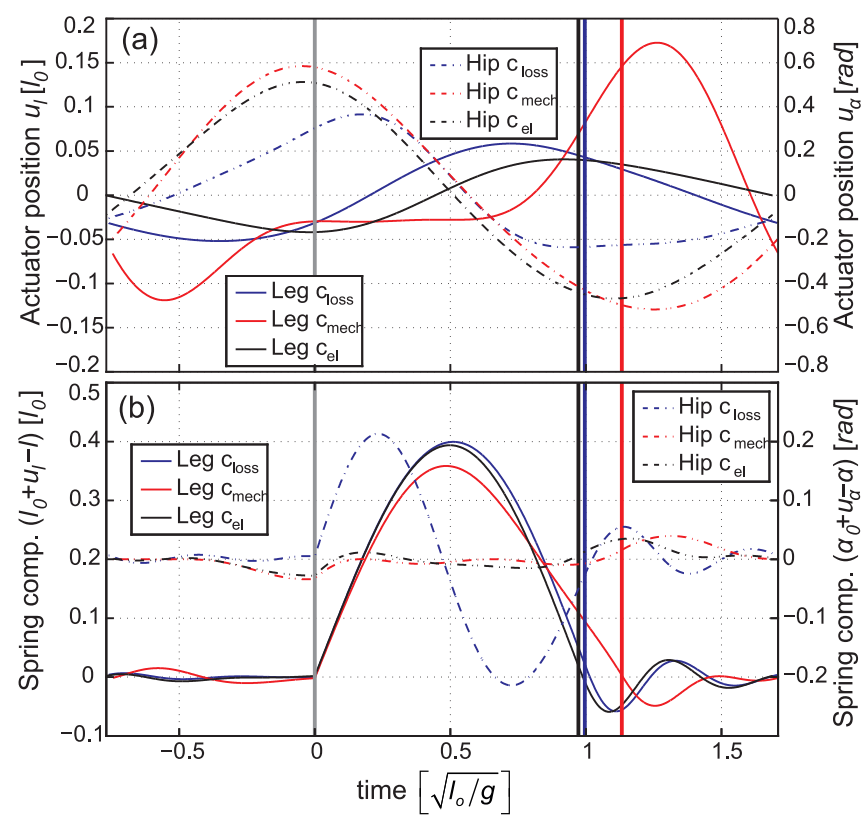

Fig. 6. The actuator profiles (a) for leg extension during forward running were qualitatively identical to those of in-place hopping. Due to increased losses, however, the amplitude of actuator motion almost doubled. The storage of energy in the hip actuator spring (b) was only exploited when minimizing $c_{\text {loss }}$, which reduced the necessary motion of the hip actuator. This allowed the selection of a higher gear ratio and reduced thermal losses.

were the capacity for shock absorbance and the reduction of peak powers.

In contrast, when optimizing for $c_{\text {loss }}$, a substantial amount of energy was stored in the hip spring (Fig. 6b). In this way, the necessary amplitude of the actuator motion and the associated accelerations could be reduced. This comes at the cost of higher spring forces, which were accommodated with a higher gear ratio. The spring forces also created a substantial reaction torque on the main body, which (with the selected inertial parameters) led to a pitching motion with an amplitude of over $0.15 \mathrm{rad}$. This is undesired and inevitable in a one-legged hopper, but could be avoided by using multiple legs and gaits in which two legs swing against each other and compensate the induced moment on the main body.

\section{COMPARISON AND CONCLUSIONS}

The presented optimization approach might be applied at various stages in the design process of a running robot. The computation of $c_{\text {mech }}$ does not require an actuator model and is thus well suited for the evaluation of conceptual design variations at a very early stage. For models that include an actuator model, $c_{\text {loss }}$ has the best convergence behavior which makes it an ideal candidate for exploring a large parameter space. The highest accuracy in predicting energetic costs is obtained by using $c_{e l}$. Especially for the planning of actual excitation trajectories, this level of precision is imperative, as the trajectories produced by different cost functions differ widely.

To be able to judge the degree to which we can transfer the

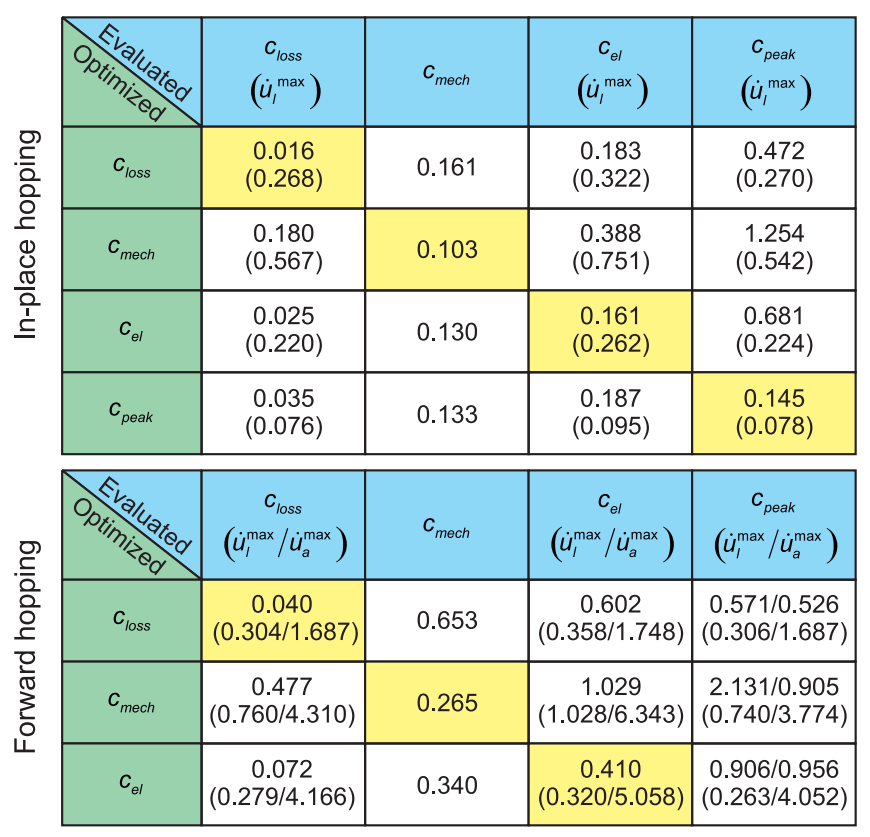

Fig. 7. Actuator profiles that were obtained when optimizing for a certain cost function (given in rows) are evaluated against the full set of cost functions (given in columns). When applicable, the maximal actuator velocity $\dot{u}_{l}^{\max }$ was obtained by optimization and is reported individually for each case in parentheses.

optimization outcome obtained using one objective function onto other scenarios, the different results are compared against each other in Fig. 7. Actuator motion profiles in (11) that were obtained by optimizing for one cost function (given in rows) were evaluated with respect to each of the other cost functions (given in columns). The power transmission parameters $\dot{u}_{l}^{\max }$ and $\dot{u}_{\alpha}^{\max }$, which were required for the evaluation of $c_{l o s s}, c_{e l}$, and $c_{\text {peak }}$, were optimized individually for each case to obtain a truly minimal value of cost. If applicable, the parameter values are reported in parentheses. The more pronounced the motion of the actuator is, the larger the maximal actuator speed was, which essentially reflects (17). When comparing in-place hopping with forward hopping, the prismatic leg action accounted for the largest part of the 2.5fold increase in cost that was observed equally for all three cost functions.

$c_{\text {mech }}$ minimizes the mechanical losses in the system and thus serves as a lower bound for the overall energy cost. The actual electrical power consumption will inevitably be higher. The magnitude of the thermal losses is approximately $15 \%$ of the positive mechanical work, if each cost function is optimized separately. Since minimizing electrical losses and minimizing mechanical losses require two distinctly different actuator profiles, the actual electrical energy consumption $c_{e l}$ exceeds $c_{m e c h}$ by about $55 \%$. One should note that the contribution of thermal losses in this case is only $15 \%$ and, in contrast to common belief, these losses do NOT dominate energy expenditure. The ratio of electrical to mechanical energy is nearly constant over a range of hopping heights from $1.1 \mathrm{l}_{\mathrm{o}}$ to $1.3 \mathrm{l}_{\mathrm{o}}$ and, in the $2 \mathrm{D}$ case, for forward speeds 

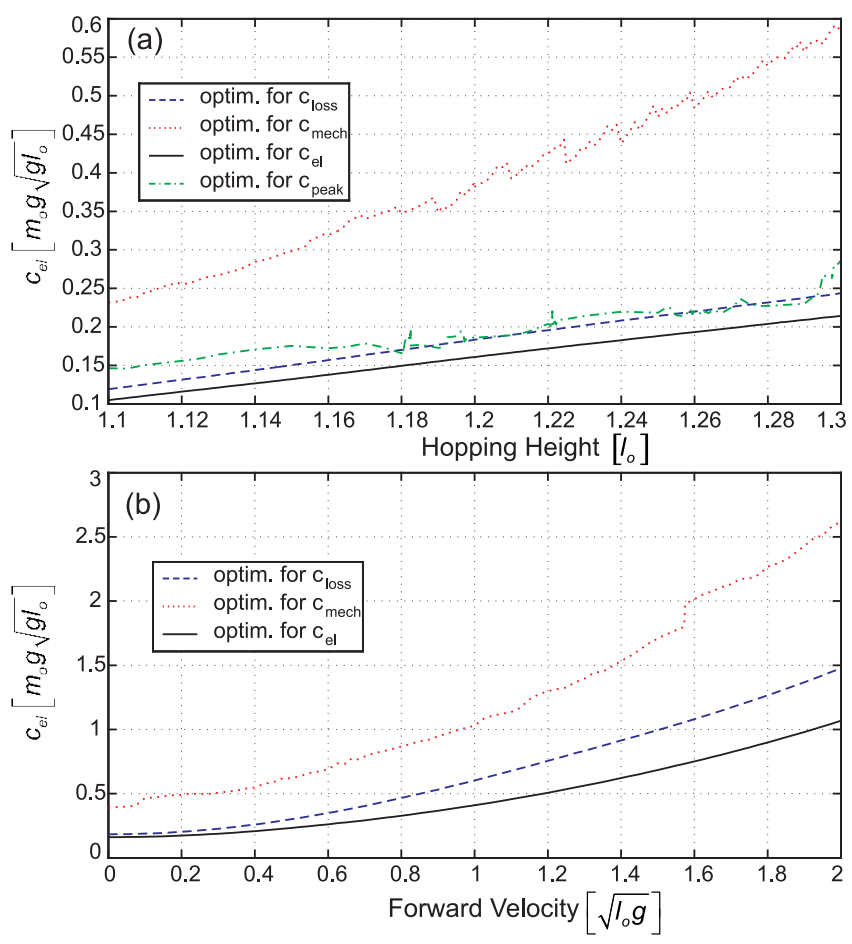

Fig. 8. Integral of positive electrical power, shown as a function of hopping height (a) and forward speed (b). Actuator profiles generated with a motor model $\left(c_{\text {loss }}, c_{e l}\right.$, and $\left.c_{\text {peak }}\right)$ show similar performances. The profile created when minimizing positive mechanical work $c_{m e c h}$ consumes about twice as much electrical energy.

up to $2 \sqrt{g_{\mathrm{o}}}$. These ratios can thus be used to estimate the electrical power consumption based on the mechanical costs.

Note that the actuator motion profile obtained when optimizing for $c_{\text {mech }}$ is not appropriate for producing actual gaits. The positive electrical work required for this actuation profile is about twice as high as for a motion optimized for $c_{e l}$ (Figure 8). In contrast, the profiles obtained with a motor model $\left(c_{\text {loss }}, c_{e l}\right.$, and $c_{\text {peak }}$ ) show only small differences in electrical work, even though the actuator profiles themselves vary considerably. For example, the actuator motion that minimizes thermal losses requires only $10 \%$ more positive electrical work than a motion that was specifically optimized for $c_{e l}$, and it can be seen in Fig. 7 by comparing the values of $c_{\text {peak }}$ in the first and third rows of the table for in-place hopping that it can be achieved with an actuator that weighs about $30 \%$ less. The opportunity to reduce mass is, of course, even more pronounced with actuator profiles that were optimized to minimize actuator mass, where the weight reduction is almost $80 \%$ in comparison to profiles that were optimized for $c_{e l}$ (while the increase in electrical energy consumption is only about $16 \%$ ).

Such a reduced actuator mass is highly beneficial, since the overall weight of the robotic system (which to a large extend is made up by the actuators) is reduced. Since all forces and powers have been expressed in normalized units as a function of the overall mass $m_{0}$, reducing this mass has a very direct impact on the power and energy consumption of an actual robot.

\section{REFERENCES}

[1] R. M. Alexander, "3 uses for springs in legged locomotion," The International Journal of Robotics Research, vol. 9, no. 2, pp. 53-61, 1990.

[2] H. Geyer, A. Seyfarth, and R. Blickhan, "Compliant leg behaviour explains basic dynamics of walking and running," Proceedings of the Royal Society B, vol. 273, no. 1603, pp. 2861-2867, 2006.

[3] A. D. Kuo, "A simple model of bipedal walking predicts the preferred speed-step length relationship," Journal of Biomechanical Engineering, vol. 123, no. 3, pp. 264-269, 2001.

[4] G. A. Lichtwark and A. M. Wilson, "In vivo mechanical properties of the human achilles tendon during one-legged hopping," Journal of Experimental Biology, vol. 208, no. 24, pp. 4715-4725, 2005.

[5] S. Mochon and T. A. McMahon, "Ballistic walking: an improved model," Mathematical Biosciences, vol. 52, no. 3-4, pp. 241-60, 1980.

[6] T. McGeer, "Passive dynamic walking," The International Journal of Robotics Research, vol. 9, no. 2, pp. 62-82, 1990.

[7] M. Hutter, C. D. Remy, and R. Siegwart, "Design of an articulated robotic leg with nonlinear series elastic actuation," in International Conference on CLimbing And WAlking Robots, CLAWAR, pp. 645652, 2009.

[8] G. Pratt and M. Williamson, "Series elastic actuators," in International Conference on Intelligent RObots and Systems, IROS, vol. 1, pp. 39906, 1995.

[9] D. W. Robinson, J. E. Pratt, D. J. Paluska, and G. A. Pratt, "Series elastic actuator development for a biomimetic walking robot," in International Conference on Advanced Intelligent Mechatronics, AIM, pp. 561-68, 1999.

[10] J. Pratt, B. Krupp, and C. Morse, "Series elastic actuators for high fidelity force control," Industrial Robot, vol. 29, no. 3, pp. 234-41, 2002.

[11] C. K. Chow and D. H. Jacobson, "Studies of human locomotion via optimal programming," Mathematical Biosciences, vol. 10, no. 3-4, pp. $239-306,1971$.

[12] V. V. Beletskii and P. S. Chudinov, "Parametric optimization in the problem of bipedal locomotion," Izv. An SSSR. Mekhanika Tverdogo Tela [Mechanics of Solids], vol. 1, pp. 25 - 35, 1977.

[13] M. Srinivasan, "Fifteen observations on the structure of energyminimizing gaits in many simple biped models," Journal of The Royal Society Interface, vol. 8, no. 54, pp. 74-98, 2011.

[14] M. Srinivasan and A. Ruina, "Computer optimization of a minimal biped model discovers walking and running," Nature, vol. 439, no. 7072 , pp. $72-75$, 2006. 10.1038/nature04113.

[15] K. Mombaur, "Using optimization to create self-stable human-like running," Robotica, vol. 27, pp. 321-30, 2009.

[16] M. Stelzer, M. Hardt, and O. von Stryk, "Efficient dynamic modeling, numerical optimal control and experimental results for various gaits of a quadruped robot," in International Conference on CLimbing And WAlking Robots, CLAWAR, 2003.

[17] M. H. Raibert, Legged robots that balance. The MIT Press series in artificial intelligence, Cambridge, Mass.: MIT Press, 1986.

[18] A. L. Hof, "Scaling gait data to body size," Gait \& Posture, vol. 4, no. 3, pp. 222-223, 1996.

[19] C. Glocker, "On frictionless impact models in rigid-body systems," Philosophical Transaction of the Royal Society A, vol. 359, no. 1789, pp. 2385-04, 2001.

[20] M. Laughton and D. Warne, eds., Electrical Engineers's Reference Book. Elsevier Science, 16 ed., 2003.

[21] P. Chatzakos and E. Papadopoulos, "The influence of dc electric drives on sizing quadruped robots," in International Conference on Robotics and Automation, ICRA, pp. 793-798, 2008.

[22] M. Hutter, C. D. Remy, M. H. Hoepflinger, and R. Siegwart, "ScarlETH: Design and control of a planarrunning robot," in International Conference on Intelligent RObots and Systems, IROS, p. (accepted for publication), 2011.

[23] O. Von Stryk, Numerische Loesung optimaler Steuerungsprobleme: Diskretiesierung, Parameteroptimierung und Berechnung der adjungierten Variablen. PhD thesis, TU München, 1994.

[24] M. Stelzer, Forward Dynamics Simulation and Optimization of Walking Robots and Humans. PhD thesis, TU Darmstadt, 2007.

[25] C. D. Remy, K. W. Buffinton, and R. Siegwart, "A matlab framework for efficient gait creation," in International Conference on Intelligent RObots and Systems, IROS, p. (accepted for publication), 2011.

[26] V. A. Tucker, "The energetic cost of moving about.," American Scientist, vol. 63, no. 4, pp. 413-149, 1975. 